\title{
Intra-operative Radiotherapy for Early Breast Cancer
}

\author{
a report by \\ Frederik Wenz, MD \\ Professor and Chairman, Department of Radiation Oncology, Mannheim Medical Center, \\ University of Heidelberg
}

DOI: $10.17925 /$ OHR.2006.00.00.41

Changing Paradigms in Loco-regional
Management of Breast Cancer

The last three decades have seen a dramatic change in the way primary tumors and lymph nodes (LNs) are managed. Breast surgery has become less radical in approach. Breast-conserving surgery (BCS) is currently the preferred operative approach, avoiding the psychological and functional consequences of radical breast amputation. Detection and selective excision of the sentinel LN has only been shown to be equivalent to radical axillary dissection while reducing operative morbidity. Breast-conserving surgery followed by whole breast radiotherapy (WBRT) with 50Gy yields equivalent local control rates compared with modified radical mastectomy, defining the current gold standard of clinical breast cancer care.

\section{Challenging the Gold Standard of WBRT}

Omission of WBRT results in in-breast tumor recurrence rates of up to $30-40 \%$ after 10 years. Nevertheless, there is a well documented underusage of WBRT after BCS, partially for reasons of patient convenience, e.g. long travelling distances to the next RT facility and assumed low risk of local recurrence. Several prospective randomized studies have therefore attempted to define patient subgroups with small, well differentiated, hormone-receptor-positive tumors in elderly patients in whom WBRT after BCS can be omitted. However, all of these studies have resulted in unacceptably high local recurrence rates, even in these highly selected patients. It was therefore concluded that all patients after BCS benefit from RT.

Realizing the clinical reality that WBRT is widely underused for the previously mentioned patients, and that BCS is not an option for a considerable number of patients due to the lack of RT facilities when radical mastectomy is the only choice, the approach of accelerated partial breast irradiation (APBI) is currently under investigation by several study groups. The concept of APBI is based on the fact that most tumor recurrences are located close to the original tumor bed and that a small subvolume of the breast may be irradiated with considerably higher single doses, therefore allowing the whole APBI to be administered in a few treatment sessions. APBI may be performed over a couple of days by external beam RT (EBRT), interstitial or intra-cavitary brachytherapy, or by a single radiation dose during intra-operative RT (IORT) using fast electrons or soft X-rays.

APBI studies include, in general, elderly patients with small, well differentiated tumors without risk factors, e.g. multifocal disease, extensive intraductal carcinoma, lymph vessel invasion, and no or minimal LN involvement. However, on the other end of the clinical spectrum, there is a subgroup of patients with a comparably high risk of local recurrence. These younger patients with larger and more aggressive tumors benefit from a local dose-escalation of RT following breastconserving surgery. The administration of the tumor bed boost may be carried out using fast electrons, interstitial brachytherapy, or IORT. IORT as a tumor bed boost has the advantage of improved targeting compared with EBRT or post-operative brachytherapy. The risk of partial or total geographic miss of the tumor bed can be minimized by this approach. Performing RT during anesthesia is certainly the most convenient sequence for the patient.

\section{IORT Using Soft X-rays}

Progress in medical technology has made mobile miniature X-ray generators, e.g. Intrabeam ${ }^{\text {TM }}$ available, which are ideally suited for IORT of breast cancer. The system is composed of a miniature X-ray source, which is small and lightweight (1.6kg), combined with a balanced floor stand with $6^{\circ}$ of freedom to gain access to target sites throughout the body (see Figure 2). This floor stand has been optimized to balance the miniature $\mathrm{X}$-ray source during positioning and treatment delivery including ergonomic features for guiding the source to the target with ease.

The miniature X-ray source has a probe with $10 \mathrm{~cm}$ length and $3.2 \mathrm{~mm}$ diameter. Within this device electrons are accelerated to the desired energy and focused down the probe to strike a gold target. This results in the production of an isotropic dose distribution of radiation around the tip of the probe. Spherical applicators ranging

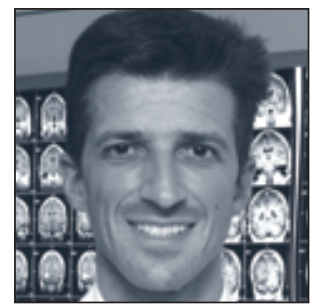

Frederik Wenz, MD, is Professor and Chairman of the Department of Radiation Oncology at the Mannheim Medical Center, University of Heidelberg. His clinical specialties are breast and prostate cancer, while his research interests are focused on novel technologies such as intensitymodulated radiotherapy (IMRT), image-guided radiotherapy (IGRT), intra-operative radiotherapy (IORT) and innovative gene-therapeutic approaches to protect normal tissues. After his board certification in radiation oncology and completion of the habilitation, he became Attending and Assistant Professor at the University of Heidelberg. In 1996, he was as a research fellow in cancer biology at the Harvard School of Public Health. Prior to that, Dr Wenz was a resident in radiation oncology at the University of Heidelberg, where he was also involved in the heavy-ion therapy project at Gesellschaft für Schwerionen-forschung $\mathrm{mbH}$ (GSI)/Darmstadt. Dr Wenz started his internship in diagnostic radiology at the German Cancer Research Center in Heidelberg/Germany after completing his studies in medicine at the University of Heidelberg, University of Birmingham, UK, University of Texas at San Antonio and Northwestern University, Chicago. 
Figure I: RT After BCS May Become More Risk-adapted in the Future
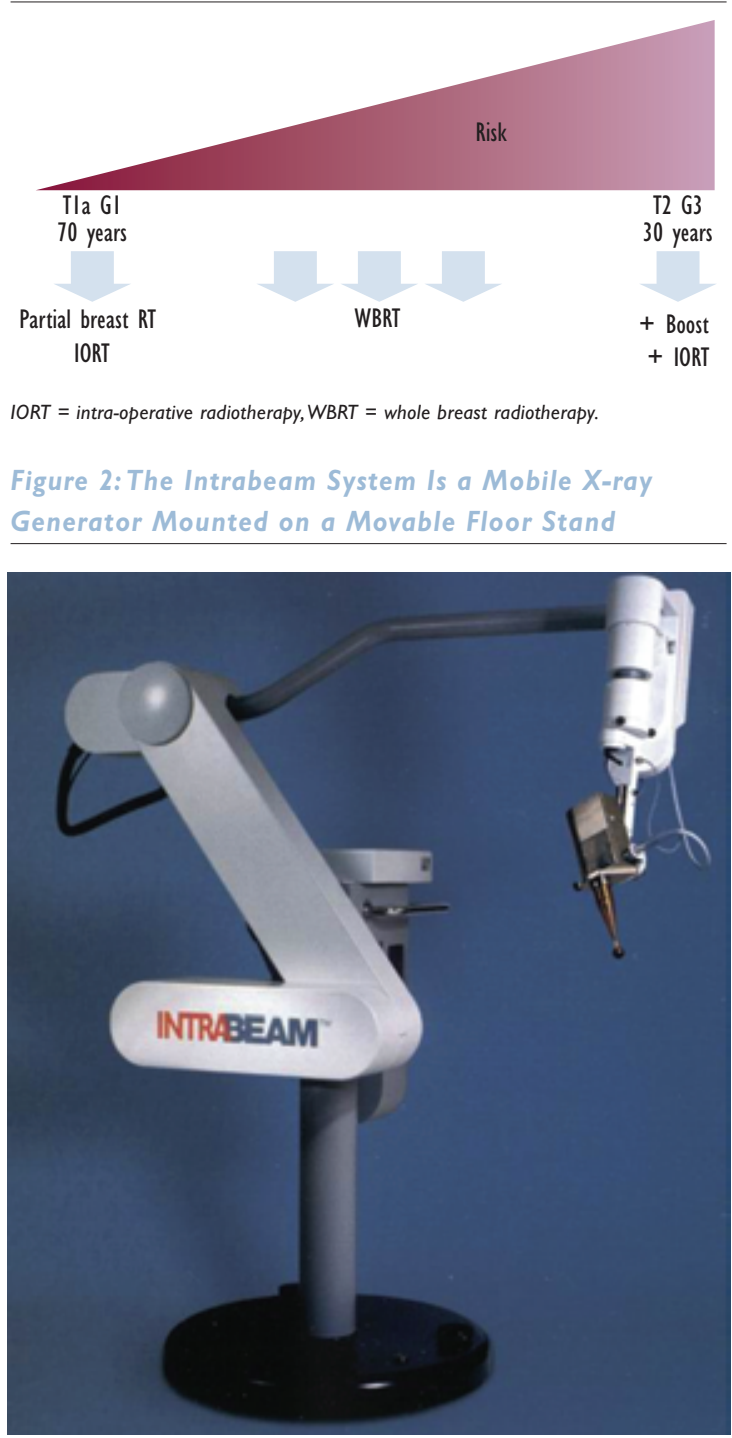

from $1.5 \mathrm{~cm}$ to $5 \mathrm{~cm}$ diameters are available. They are fixed at the end of the source and located in the excision cavity (see Figure 3), achieving a homogeneous dose distribution on the applicators' surface and, consequently, the surface of the tumor cavity.

The X-ray system produces low-energy photons $(30-50 \mathrm{Kv})$ with a steep dose fall-off in soft tissue; therefore, no special shielding is required in the room and the treatment can be carried out in unmodified operating theatres with minimal exposition of patient and staff. The rapid depth dose fall-off in the tissue around the applicator allows a minimal exposure of the surrounding tissue such as in cases of breast cancer and lung and cardiac tissue.

The dose is prescribed in the author's institution at the surface of the tumor cavity that corresponds to the applicator surface. The treatment time depends on the chosen applicator size.
After surgical tumor removal, an appropriate spherical applicator is placed into the tumor bed, depending on the size of the surgical cavity. Intrabeam is then completely wrapped in a sterile plastic cover with a hole for the sterile applicator to pass through. Before the final fixation of the applicator, a purse-string suture is made within the mobilized breast tissue in order to bring the target tissue in close contact with the applicator surface. If source and applicator are placed in the tumor cavity, the purse-string is tightened. After that, the skin is everted by one or two distracters to avoid that skin tissue is less than $1 \mathrm{~cm}$ from the applicator surface. The control console has a weight of $18 \mathrm{~kg}$ and length, width, and height of $47 \mathrm{~cm}, 39.4 \mathrm{~cm}$ and $20.3 \mathrm{~cm}$, respectively. After the treatment, the applicator is carefully removed and the wound is closed as usual. The operating time is prolonged by the IORT for 20-50 minutes.

\section{The Current and Future Role of IORT for Breast Cancer}

The current gold standard of care for early operable breast cancer is breast conserving surgery followed by post-operative RT to the whole breast.

Due to more effective screening methods, breast cancers are found more frequently in very early stages; the question arises whether all patients have to be uniformly treated by WBRT. Since local recurrences after breastconserving surgery occur mainly in the area around the original primary tumor, localized RT delivered to the peri-tumoral tissue as accelerated partial breast irradiation (APBI) could be an appropriate method to control local recurrence in selected patients with early-stage breast cancer. Several studies investigating this approach are under way. No final conclusion in terms of survival and equivalence to the standard six-week post-operative RT can be drawn to date, since the follow-up and the number of patients are limited. Only the results of the multicentric randomized trials such as Targeted Intraoperative Radiotherapy (TARGIT), Electron Intraoperative Treatment (ELIOT), or the National Surgical Adjuvant Breast and Bowel Project (NSABP) trial designed to test the hypothesis of equivalence between the two treatment arms will give appropriate answers. It is expected that the first results of these trials will be available in 2007 .

IORT as a local tumor bed boost is a valid alternative to the boost by EBRT using fast electrons or by interstitial brachytherapy. The risk of geographic miss is minimized, skin protection can be easily achieved, and the overall treatment time is shortened. Long-term results demonstrating effectivity and good cosmetic results have already been presented by several groups.

In-breast tumor recurrence or second primaries in the same breast years after BCS and WBRT are rare 
but clinically challenging events. Due to the limited tolerance of the breast to radiation, a second course of EB-WBRT is not possible. Most patients therefore undergo radical mastectomy, even for small recurrent tumors or second primaries. IORT may become a valid option for these patients allowing a second local tumor excision and intra-operative delivery of a focal high dose of radiation.

\section{Conclusion}

There has been a paradigm shift in the surgical approach of breast cancer over the last three decades with a considerable risk-adapted reduction in radicality as to the breast and the LNs. Currently, the dogma of WBRT with 50Gy for all patients after breast conserving surgery (BCS) is challenged. There is a subgroup of patients who have a clear benefit from a local dose escalation to the tumor bed.

On the other hand, there may be a subgroup of patients for whom irradiating the tumor bed by accelerated partial breast irradiation (APBI) may become an option pending the results of the on-going studies. IORT is a method for boost and APBI and will most likely gain acceptance in the near future.
Figure 3: For IORT of Breast Cancer, a Suitable

Spherical Applicator is Used for Homogenous

Irradiation of the Excision Cavity

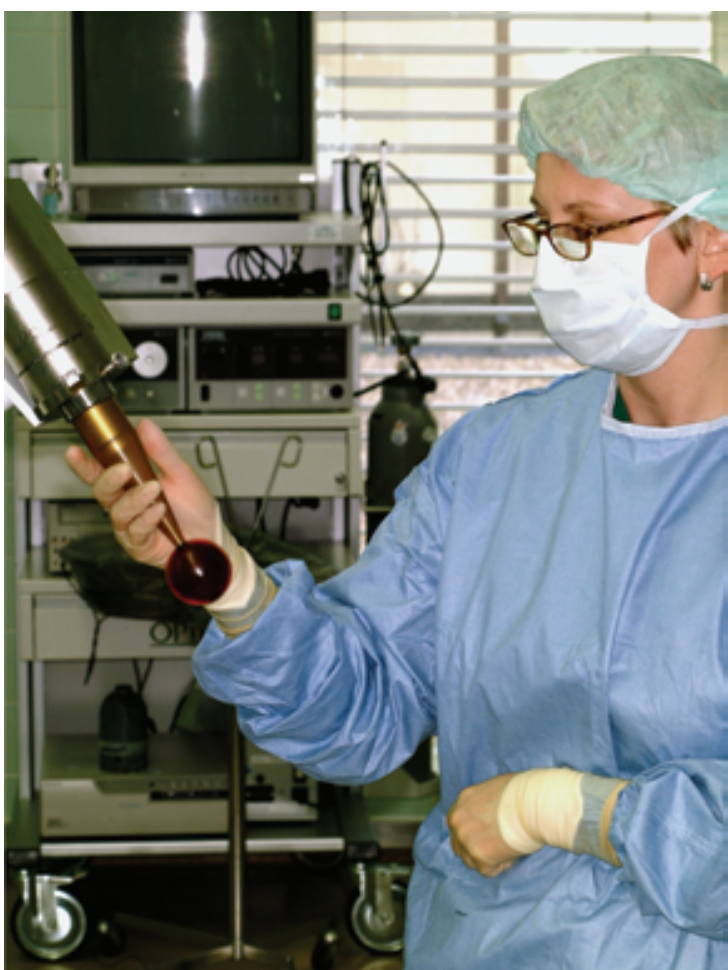

TAREET: abbreviated partial breast irradiation in one fraction. Intraoperative radiotherapy with the INTRABEAM ${ }^{\circ}$ system.

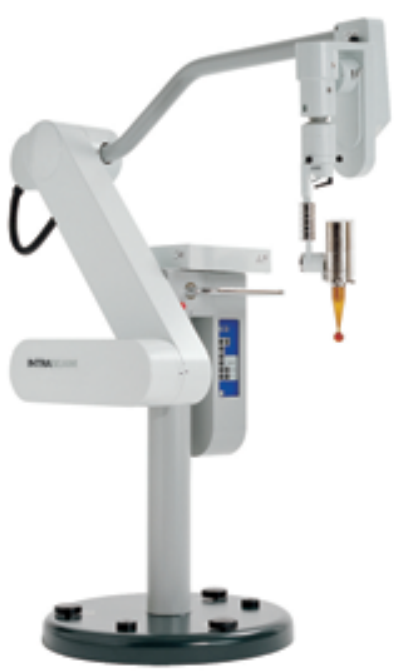

Carl Zeiss Surgical Inc.

One Zeiss Drive

Thornwood, NY 10594
Phone 914-747-1800

Fax 914-682-8296

www.zeiss.com/radiotherapy 